\title{
The 3-D structure of the Coma-A 1367 supercluster: Optical spectroscopy of 102 galaxies $^{\star}$
}

\author{
G. Gavazzi ${ }^{1}$, L. Carrasco ${ }^{2,3}$, and R. Galli ${ }^{1}$ \\ 1 Universitá degli Studi di Milano, Via Celoria 16, 20133 Milano, Italy \\ 2 Instituto Nacional de Astrofísica, Optica y Electrónica, Apartado Postal 51, C.P. 72000 Puebla, Pue., Mexico \\ 3 Observatorio Astronómico Nacional/UNAM, Ensenada B.C., Mexico
}

Received July 3; accepted October 2, 1998

\begin{abstract}
Optical spectroscopy of 117 CGCG galaxies, 102 of which are projected in the direction of the ComaA 1367 supercluster, is reported. These new measurements, added to those found in the literature, bring to 1068 the number of CGCG galaxies in this region with available redshift, out of a total of 1085 objects with $m_{\mathrm{p}} \leq 15.7$. We use these data to infer the 3 -D structure of the Coma supercluster.
\end{abstract}

Key words: galaxies: redshift — galaxies: clusters: Coma; A 1367

\section{Introduction}

The Coma-A 1367 supercluster is part of the Great Wall, the major large-scale structure in the Northern Hemisphere (Zabludoff et al. 1993). This supercluster provides us with a unique test-bed for extragalactic investigations since it contains thousands relatively bright galaxies $\left(m_{\mathrm{p}}<15.7\right)$ at high galactic latitude, thus little affected by extinction from our Galaxy, distributed in a variety of environments spanning from rich clusters (Coma + A 1367) to relatively low-density regions. Furthermore, due to its narrow distribution in the redshift space relative to its average recessional velocity $\left(7000 \mathrm{~km} \mathrm{~s}^{-1}\right)$, the distance spread of its members is minimal. These properties make the Coma supercluster ideal for studying the environmental dependence of the properties of galaxies (e.g. their luminosity function) spanning a local density regime of over an order of magnitude. This structure has been studied in increasing details in the last

Send offprint requests to: gavazzi@uni.mi.astro.it

* Based on observations obtained with the Loiano telescope belonging to the University of Bologna, Italy, and with the G. Haro telescope of the INAOE, Mexico. decades, as new redshift measurements became available. The pioneering work of Gregory \& Thompson (1978) unveiled the main structure of the supercluster within the region $11^{\mathrm{h}} 30^{\mathrm{m}}<\alpha<13^{\mathrm{h}} 30^{\mathrm{m}} ; 18^{\circ}<\delta<32^{\circ}$. By that time only 238 of the 1130 galaxies brighter than 15.7 catalogued in the CGCG (Zwicky et al. 1961-68) in this region, had known redshift values. Ten years later the analysis of Gavazzi (1987) counted more than twice as many velocity measurements (695) obtained from optical (de Lapparent et al. 1986; Huchra et al. 1990) and $21 \mathrm{~cm}$ spectra (Giovanelli \& Haynes 1985). The recent determination of the luminosity function of galaxies in this region by Gavazzi et al. (1995) relied on 941 redshifts. However complete optical and near-IR photometry was available for the late-type population only (Gavazzi \& Boselli 1996). Since then the photometric survey was extended to cover the early-type galaxies as well, at least in the near-IR (Gavazzi et al. in preparation), while measurements in the visible are still under way. The full exploitation of this photometric material requires extending the redshift survey to the remaining 189 unmeasured galaxies. For this purpose we undertook the spectroscopic survey presented in this paper. The sample is presented in Sect. 2, the observations and data reduction in Sect. 3. The new redshift are given in Sect. 4 and are used, joint with the measurements from the literature, to study the $3-\mathrm{D}$ structure of the Coma supercluster.

\section{The sample}

Galaxies in the present study are selected from the CGCG Catalogue (Zwicky et al. 1961-68) in the region $11^{\mathrm{h}} 30^{\mathrm{m}}<$ $\alpha<13^{\mathrm{h}} 30^{\mathrm{m}} ; 18^{\circ}<\delta<32^{\circ}$ containing the Coma-A 1367 supercluster. There are 1130 galaxies listed in this region. Of these 1085 have $m_{\mathrm{p}} \leq 15.7$. Another 45 belong to multiple systems which were split in their individual components, each of them fainter than the catalogue limiting magnitude 15.7. Out of the 1130 we observed 102 galaxies 
Table 1. The spectrograph characteristics

\begin{tabular}{cccccc}
\hline Tel & Spectrog. & $\begin{array}{c}\text { dispersion } \\
\AA / \mathrm{mm}\end{array}$ & $\begin{array}{c}\text { coverage } \\
\AA\end{array}$ & CCD type & $\begin{array}{c}\text { pix } \\
\mu \mathrm{m}\end{array}$ \\
\hline Loiano & BFOSC & 198 & $4060-7900$ & $1024 \times 1024 \mathrm{TH}$ & 19 \\
Cananea & LFOSC & 228 & $4000-7100$ & $576 \times 384 \mathrm{TH}$ & 23 \\
\hline
\end{tabular}

with no $z$ values available in the literature. Only $17 / 1085$ galaxies with $m_{\mathrm{p}} \leq 15.7$ in the Coma region and 45/1130 fainter than this limit remain with unknown redshift, thus the sample is $98 \%$ complete at 15.7 . Spectra of 3 galaxies belonging to the cluster A 262 and 12 to the Hercules supercluster were also taken as fillers.

\section{Observations and data reduction}

Low dispersion spectra of 117 galaxies were obtained in several observing runs since 1995 using the imaging spectrographs BFOSC and LFOSC attached to the Cassini $1.5 \mathrm{~m}$ telescope (Loi) at Loiano (Italy) and the $2.1 \mathrm{~m}$ telescope (Can) of the Guillermo Haro Observatory at Cananea (Mexico), respectively.

Table 1 lists the characteristics of the two spectrographs in the adopted configurations:

The observations at Loiano were performed using a 2.0 or 2.5 arcsec slit, depending on the seeing conditions, oriented E-W. The exposure time ranged between 20 and 90 min according to the brightness of the target object. Observations at Cananea were carried out with a 3.1 arcsec fixed slit, oriented N-S. Every galaxy spectrum was preceded and followed by an exposure of a HeAr lamp (Loiano) or XeNe lamp (Cananea) to secure the wavelength calibration.

Data reduction was performed in the IRAF-PROS environment. After bias subtraction, when 3 or more frames of the same target were obtained, these were combined (after spatial alignment) using a median filter to help cosmic rays removal. Otherwise the cosmic rays were removed under visual inspection. The wavelength calibration was checked on known sky lines. These were found within $\sim 1 \AA$ from their nominal value, providing an estimate of the systematic uncertainty on the derived velocities of $\sim 50 \mathrm{~km} \mathrm{~s}^{-1}$. After subtraction of the sky background, one-dimensional spectra were extracted from the frames. These spectra were analyzed with either of two methods:

1) individual line measurement: all spectra taken at Cananea and those obtained at Loiano prior to 1996 were inspected and emission/absorption lines were identified. Emission lines include $\mathrm{H} \alpha, \mathrm{N}[\mathrm{II}]$ and $\mathrm{S}[\mathrm{II}]$. Absorption lines include the $\mathrm{Mg}[\mathrm{I}], \mathrm{CaFe}$ and $\mathrm{Na}$. The galaxy redshift was obtained from these individual measurements. If more than one line was identified, the galaxy redshift was derived as the weighted mean of the individual measurements, with weights proportional to the line intensities.

2) Cross correlation technique: spectra obtained at Loiano after 1996 were analyzed using the crosscorrelation technique of Tonry \& Davis (1979). This method is based on a "comparison" between the spectrum of a galaxy whose redshift is to be determined, and a fiducial spectral template of a galaxy (or star) of appropriate spectral type to contain the wanted absorption/emission lines. The basic assumption behind this method is that the spectrum of a galaxy is well approximated by the spectrum of its stars, modified by the effects of the stellar motions inside the galaxy and by the systemic redshift. For this purpose high signal-tonoise spectra were taken of two template galaxies: M105 (absorption lines) and NGC 1330 (emission lines), whose redshifts are 866 and $1039 \mathrm{~km} \mathrm{~s}^{-1}$ respectively.

The observed redshifts $\left(V_{\text {obs }}\right)$ were first transformed to Heliocentric $\left(V_{\mathrm{hel}}\right)$, then corrected for the motion of our galaxy relative to the Cosmic Microwave Background $\left(V_{\mathrm{CMB}}\right)$ according to Kogut et al. (1993).

\section{Results}

The velocity measurements obtained in this work are listed in Table 2 as follow:

Column 1: the CGCG designation (Zwicky et al. 1961-68). Columns 2, 3: (B1950) celestial coordinates, measured with few arcsec uncertainty.

Column 4: the photographic magnitude (Zwicky et al. 1961-68).

Columns 5, 6: the derived heliocentric velocity with uncertainty. The latter quantity is obtained by adding in quadrature systematic and statistical errors.

Column 7: an asterisk marks uncertain redshifts. These are either low signal spectra or spectra with only one absorption line that could be misidentified.

Column 8: type of lines $(\mathrm{A}=$ absorption; $\mathrm{E}=$ emission; $\mathrm{EA}=$ both).

Column 9: method used for the redshift measurement $(\mathrm{CC}=$ cross correlation; IL $=$ individual line measurement).

Column 10: observing run.

Figure 1 gives a representation in celestial coordinates of 1085 galaxies (panel a) and a wedge diagram $\left(V_{\mathrm{CMB}}\right.$ vs. R.A.) is given in panel b. Small symbols mark galaxies 
Table 2. Parameters of the observed galaxies

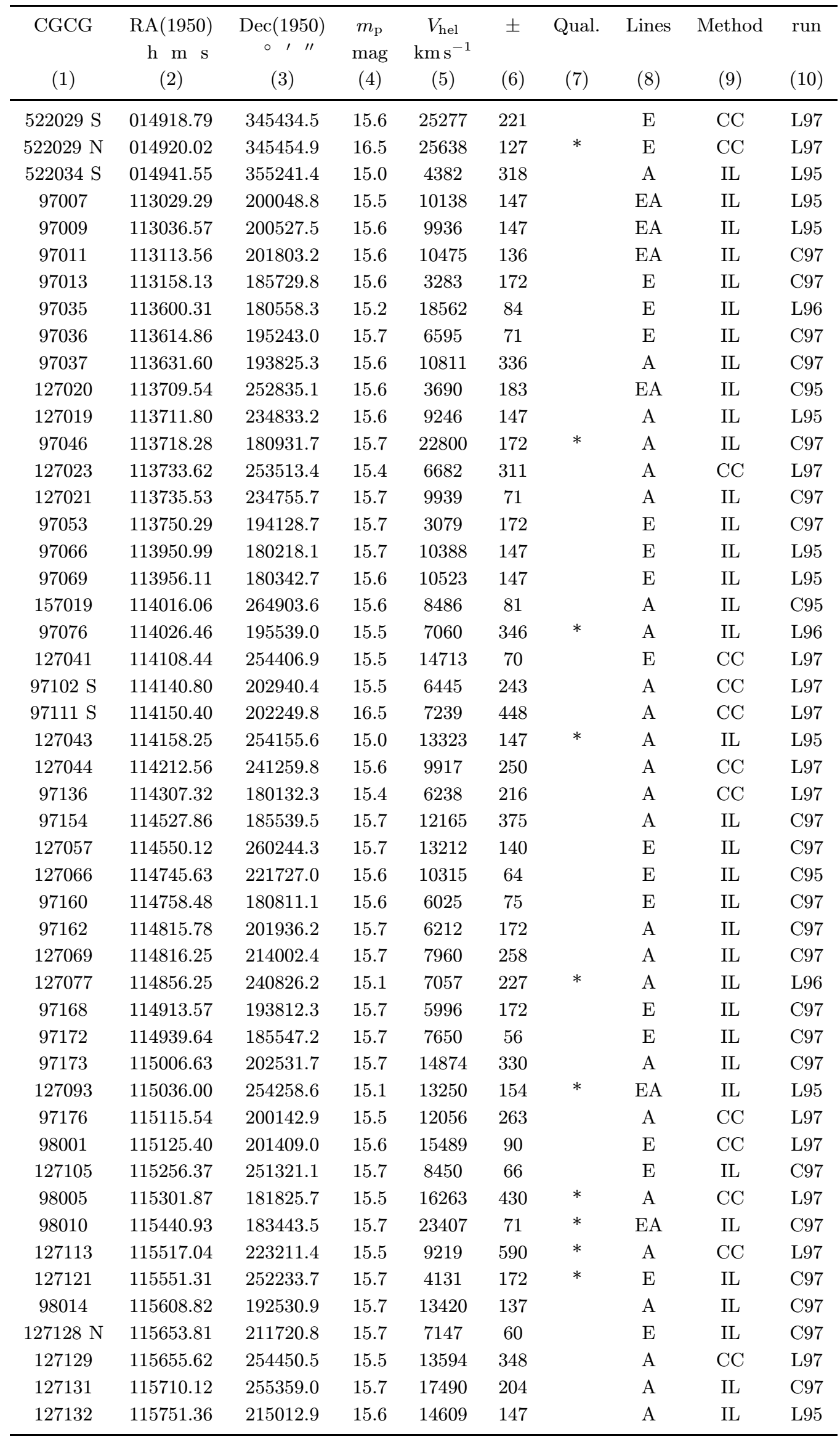


Table 2. continued

\begin{tabular}{|c|c|c|c|c|c|c|c|c|c|}
\hline $\begin{array}{c}\text { CGCG } \\
(1)\end{array}$ & $\begin{array}{c}\mathrm{RA}(1950) \\
\mathrm{h} \mathrm{m} \mathrm{s} \\
(2)\end{array}$ & $\begin{array}{c}\operatorname{Dec}(1950) \\
\circ, \quad " \\
(3)\end{array}$ & $\begin{array}{c}m_{\mathrm{p}} \\
\operatorname{mag} \\
(4)\end{array}$ & $\begin{array}{c}V_{\text {hel }} \\
\mathrm{km} \mathrm{s}^{-1} \\
(5)\end{array}$ & $\begin{array}{l} \pm \\
(6)\end{array}$ & $\begin{array}{c}\text { Qual. } \\
(7)\end{array}$ & $\begin{array}{c}\text { Lines } \\
(8)\end{array}$ & $\begin{array}{c}\text { Method } \\
\text { (9) }\end{array}$ & $\begin{array}{l}\text { run } \\
(10)\end{array}$ \\
\hline 98018 & 115801.76 & 181752.7 & 15.7 & 6959 & 172 & $*$ & $\mathrm{E}$ & IL & C97 \\
\hline 127134 & 115838.73 & 225342.5 & 15.7 & 8439 & 110 & & $\mathrm{E}$ & IL & C97 \\
\hline 98023 & 115910.69 & 181036.1 & 15.1 & 6920 & 81 & & EA & IL & L95 \\
\hline 127138 & 115921.82 & 210134.4 & 15.5 & 7210 & 339 & $*$ & $\mathrm{~A}$ & $\mathrm{CC}$ & L97 \\
\hline $127137 \mathrm{E}$ & 115923.50 & 204057.7 & 15.6 & 22478 & 198 & & $\mathrm{~A}$ & IL & C97 \\
\hline 98026 & 115931.25 & 181127.1 & 15.6 & 6764 & 140 & & EA & IL & C97 \\
\hline 98028 & 115931.81 & 180603.3 & 15.0 & 6888 & 115 & & $\mathrm{~A}$ & IL & L96 \\
\hline 127140 & 115937.08 & 243440.5 & 15.7 & 13361 & 636 & * & $\mathrm{A}$ & IL & C97 \\
\hline 127141 & 115952.75 & 240548.8 & 15.0 & 6700 & 145 & & A & IL & L96 \\
\hline 128001 & 120003.94 & 212618.1 & 15.5 & 7489 & 206 & & $\mathrm{~A}$ & $\mathrm{CC}$ & L97 \\
\hline 128006 & 120127.50 & 203656.6 & 15.6 & 6719 & 100 & & A & IL & C97 \\
\hline 98045 & 120140.37 & 202917.1 & 15.6 & 6375 & 125 & & $\mathrm{~A}$ & IL & C97 \\
\hline 128011 & 120143.20 & 242518.0 & 15.7 & 15073 & 160 & $*$ & $\mathrm{~A}$ & IL & C97 \\
\hline 98048 & 120217.37 & 193134.0 & 15.2 & 6944 & 206 & & $\mathrm{~A}$ & IL & L96 \\
\hline 128015 & 120222.87 & 213109.7 & 15.3 & 6832 & 147 & & $\mathrm{E}$ & IL & L95 \\
\hline 128019 & 120254.50 & 203511.9 & 15.0 & 7289 & 224 & & $\mathrm{EA}$ & IL & L96 \\
\hline 128018 & 120256.06 & 203130.7 & 15.1 & 7257 & 137 & & A & IL & L96 \\
\hline 128020 & 120304.19 & 205001.9 & 14.9 & 7155 & 140 & & $\mathrm{~A}$ & IL & L96 \\
\hline 128022 & 120307.00 & 205002.3 & 15.2 & 7580 & 71 & $*$ & $\mathrm{~A}$ & IL & L96 \\
\hline 128024 & 120318.31 & 204759.0 & 15.3 & 7268 & 316 & & A & $\mathrm{CC}$ & L97 \\
\hline 98054 & 120344.04 & 182850.2 & 15.6 & 6868 & 239 & & $\mathrm{~A}$ & IL & C97 \\
\hline 98067 & 120607.30 & 181404.4 & 15.7 & 7610 & 172 & & $\mathrm{E}$ & IL & C97 \\
\hline 98068 & 120633.95 & 202705.2 & 15.7 & 19324 & 976 & & $\mathrm{~A}$ & IL & C97 \\
\hline 98072 & 120658.36 & 192915.9 & 15.7 & 7946 & 172 & & $\mathrm{E}$ & IL & C97 \\
\hline 98074 & 120725.28 & 201146.0 & 15.6 & 7471 & 147 & & $\mathrm{E}$ & IL & L95 \\
\hline 98075 & 120732.92 & 202931.7 & 15.7 & 24934 & 172 & $*$ & $\mathrm{~A}$ & IL & C97 \\
\hline 98076 & 120741.87 & 192818.1 & 15.5 & 7804 & 385 & & $\mathrm{~A}$ & $\mathrm{CC}$ & L97 \\
\hline 98081 & 120809.37 & 180955.8 & 15.2 & 7177 & 132 & & EA & IL & L95 \\
\hline 98082 & 120812.94 & 191907.8 & 14.7 & 7454 & 76 & & $\mathrm{~A}$ & IL & L96 \\
\hline 98087 & 120849.31 & 192200.4 & 15.3 & 7540 & 122 & & $\mathrm{~A}$ & IL & L95 \\
\hline 98090 & 120905.37 & 200724.7 & 15.7 & 6537 & 99 & & $\mathrm{E}$ & $\mathrm{CC}$ & L98 \\
\hline 98091 & 120911.77 & 194606.1 & 15.6 & 3350 & 92 & & $\mathrm{E}$ & $\mathrm{CC}$ & L98 \\
\hline 98104 & 121045.94 & 201249.3 & 15.6 & 8349 & 147 & & $\mathrm{E}$ & IL & L95 \\
\hline 98112 & 121135.86 & 193650.2 & 15.6 & 15198 & 400 & & $\mathrm{~A}$ & $\mathrm{CC}$ & L97 \\
\hline 128057 & 121150.34 & 222755.4 & 15.6 & 6998 & 174 & $*$ & $\mathrm{E}$ & $\mathrm{CC}$ & L97 \\
\hline 98121 & 121343.01 & 180441.8 & 15.4 & 9000 & 153 & & $\mathrm{E}$ & IL & L95 \\
\hline 98122 & 121344.64 & 184125.4 & 15.7 & 8968 & 125 & & $\mathrm{E}$ & $\mathrm{CC}$ & L98 \\
\hline 99002 & 121523.76 & 184037.5 & 15.5 & 7681 & 108 & & $\mathrm{E}$ & $\mathrm{CC}$ & L97 \\
\hline 128072 & 121536.82 & 245758.1 & 15.4 & 6866 & 69 & & $\mathrm{E}$ & IL & C95 \\
\hline 99004 & 121543.32 & 201717.4 & 15.6 & 8744 & 138 & & $\mathrm{E}$ & IL & C95 \\
\hline 99006 & 121552.00 & 202510.2 & 15.5 & 8516 & 94 & & $\mathrm{E}$ & $\mathrm{CC}$ & L97 \\
\hline 99009 & 121609.64 & 182819.2 & 15.6 & 9358 & 166 & & $\mathrm{E}$ & $\mathrm{CC}$ & L98 \\
\hline 128076 & 121717.15 & 210706.0 & 15.7 & 9359 & 78 & & $\mathrm{E}$ & $\mathrm{CC}$ & L98 \\
\hline 128086 & 122227.12 & 243933.5 & 15.6 & 10245 & 311 & $*$ & EA & $\mathrm{CC}$ & L98 \\
\hline 128088 & 122337.28 & 203646.4 & 15.7 & 9361 & 385 & $*$ & $\mathrm{~A}$ & $\mathrm{CC}$ & L98 \\
\hline 128091 & 122601.19 & 233452.2 & 15.3 & 6639 & 278 & & $\mathrm{~A}$ & $\mathrm{CC}$ & L97 \\
\hline
\end{tabular}

taken from the literature, filled circles mark the measurements obtained in this work.

Figure 2 gives a histogram of the galaxy velocity distribution (panel a). The present measurements are given in a separate histogram (panel b). Since, for obvious reasons, we measured galaxies fainter than average, the fraction of background objects (panel c) is higher than in the general sample. However, even at the present limiting magnitude, nearly $50 \%$ of the new redshifts are found in the range $6000-8500 \mathrm{~km} \mathrm{~s}^{-1}$, typical of the Coma supercluster.

\subsection{The 3-D structure of the Coma-A 1367 supercluster}

The aim of this work is to try an objective determination of the "aggregation" state of galaxies in the 
Table 2. continued

\begin{tabular}{|c|c|c|c|c|c|c|c|c|c|}
\hline $\begin{array}{c}\text { CGCG } \\
(1)\end{array}$ & $\begin{array}{c}\mathrm{RA}(1950) \\
\mathrm{h} \mathrm{m} \mathrm{s} \\
(2)\end{array}$ & $\begin{array}{c}\operatorname{Dec}(1950) \\
\circ \quad, \quad \\
(3)\end{array}$ & $\begin{array}{c}m_{\mathrm{p}} \\
\operatorname{mag} \\
(4)\end{array}$ & $\begin{array}{c}V_{\text {hel }} \\
\mathrm{km} \mathrm{s}^{-1} \\
(5)\end{array}$ & $\begin{array}{l} \pm \\
(6)\end{array}$ & $\begin{array}{c}\text { Qual. } \\
\text { (7) }\end{array}$ & $\begin{array}{c}\text { Lines } \\
(8)\end{array}$ & $\begin{array}{c}\text { Method } \\
\text { (9) }\end{array}$ & $\begin{array}{l}\text { run } \\
(10)\end{array}$ \\
\hline 99064 & 122605.12 & 194449.1 & 15.2 & 13497 & 203 & & EA & IL & L95 \\
\hline 129004 & 122719.12 & 223853.9 & 15.2 & 6847 & 71 & & $\mathrm{E}$ & IL & L95 \\
\hline 99070 & 122756.34 & 190508.5 & 15.4 & 14575 & 155 & & $\mathrm{E}$ & $\mathrm{CC}$ & L97 \\
\hline 99071 & 122759.12 & 195658.3 & 15.0 & 885 & 197 & * & A & $\mathrm{CC}$ & L97 \\
\hline 129006 & 122911.59 & 204525.8 & 15.6 & 1921 & 55 & & $\mathrm{E}$ & IL & C95 \\
\hline 129007 & 123232.27 & 260318.0 & 15.7 & 8673 & 114 & & $\mathrm{E}$ & IL & C97 \\
\hline $159049 \mathrm{~S}$ & 123756.90 & 264641.0 & 15.7 & 6330 & 172 & & A & IL & C97 \\
\hline $159049 \mathrm{~N}$ & 123757.20 & 264755.0 & 15.4 & 7280 & 132 & * & $\mathrm{E}$ & IL & C97 \\
\hline 161050 & 132402.10 & 315731.0 & 15.6 & 14989 & 136 & & $\mathrm{E}$ & $\mathrm{CC}$ & L98 \\
\hline $161061 \mathrm{~S}$ & 132554.00 & 285542.1 & 15.6 & 11247 & 71 & & $\mathrm{E}$ & IL & C95 \\
\hline 108024 & 155546.36 & 152733.6 & 15.6 & 11070 & 156 & & EA & IL & C97 \\
\hline 108025 & 155605.62 & 181322.0 & 15.6 & 13481 & 74 & & A & IL & C97 \\
\hline 108032 & 155647.22 & 195309.8 & 15.6 & 5488 & 74 & & $\mathrm{E}$ & IL & L96 \\
\hline 108040 & 155807.52 & 193451.4 & 15.7 & 13817 & 147 & & $\mathrm{E}$ & IL & L96 \\
\hline 108052 & 155842.70 & 184514.5 & 15.7 & 10649 & 171 & & A & IL & C97 \\
\hline 108077 & 160013.97 & 144134.3 & 15.7 & 10279 & 100 & & A & IL & C97 \\
\hline 108080 & 160034.69 & 195530.1 & 15.7 & 1663 & 188 & * & A & IL & C97 \\
\hline 108090 & 160137.46 & 150218.2 & 15.6 & 10779 & 113 & & A & IL & C97 \\
\hline 108103 & 160227.38 & 163925.9 & 15.6 & 13418 & 147 & & EA & IL & C97 \\
\hline 108114 & 160303.88 & 155219.7 & 15.7 & 10723 & 94 & & $\mathrm{E}$ & IL & L96 \\
\hline 108125 & 160319.47 & 163936.0 & 15.6 & 4603 & 56 & & $\mathrm{E}$ & IL & C97 \\
\hline $224037 \mathrm{~S}$ & 162647.90 & 411609.6 & 15.5 & 9489 & 72 & & A & IL & C97 \\
\hline
\end{tabular}

Coma-A 1367 supercluster, i.e. to establish the membership of galaxies to structures of a given size and complexity (e.g. clusters, groups, multiplets etc.) or their degree of "isolation". This is not a trivial exercise since this definition depends on two arbitrary quantities: the scale on which the local density is computed and the local density itself. Consider for example a pair of galaxies with a separation of say $100 \mathrm{kpc}$. This doublet could be relatively isolated in space, or embedded in a group of galaxies, or in a much larger cluster containing hundreds of galaxies. These three cases, in spite of their similar local densities (i.e. computed on $100 \mathrm{kpc}$ radius), are subject to extremely different environmental conditions: the first (isolated pair) is dominated by the gravitational (tidal) force induced by its companion, whereas in the second example the prevailing conditions are dictated by the group potential and in the third by the large-scale cluster potential or might be severely influenced by the diffuse intracluster gas (e.g. ram pressure). These prevailing environmental conditions are thus "described" by a density parameter which must be computed within a radius similar to the typical scale of the aggregate. Once the densities are computed, these can be converted into (mutually exclusive) "aggregation" classes using appropriate density thresholds.

\subsection{The 3-D algorithm}

The structure of the Coma-A 1367 supercluster stands out clearly in Figs. 1 and 2 as the pronounced density enhancement centered at $7200 \mathrm{~km} \mathrm{~s}^{-1}$, stretching through the entire R.A. window. Conspicuous features are: the "fingers of god" of the two clusters Coma and A 1367 which span the interval $4000<V<10000 \mathrm{~km} \mathrm{~s}^{-1}$ and the large "void" in front of the supercluster. The remaining objects, belonging to the bridge between the two clusters have a narrower velocity distribution, which lies within the interval of roughly $6000<V<8500 \mathrm{~km} \mathrm{~s}^{-1}$.

To reconstruct the 3 -D structure of the supercluster we proceed as follows:

1) Clusters (Agg $=1$ to 4$)$ : we identify the members of the two rich clusters using a preliminary conservative positional criterion: in the velocity range $4000<V<$ $10000 \mathrm{~km} \mathrm{~s}^{-1}$ there are 72 galaxies within $1 \mathrm{deg}$ radius $(\operatorname{Agg}=1)$ and 177 within $2 \mathrm{deg}(\operatorname{Agg}=2)$ of the Coma cluster and 59 galaxies within $0.5(\mathrm{Agg}=3.0)$ and 81 within 1 deg radius of A $1367(\mathrm{Agg}=4.0)$. We subtract these objects from the sample.

2) "Homunculus" ( $\mathrm{Agg}=16)$ : we identify on a purely positional basis the members of the "legs of the homunculus" (see de Lapparent et al. 1986) $(\operatorname{Agg}=16)$ and we subtract these objects from the sample.

3) Foreground (Agg $=8$ to 18$)$ : we subtract from the remaining sample all galaxies with $V<6000 \mathrm{~km} \mathrm{~s}^{-1}$, which are considered as foreground objects. These are empirically assigned to individual groups and structures on the basis of their 3 -D coordinates ( $\mathrm{Agg}=8$ to 18 as defined 


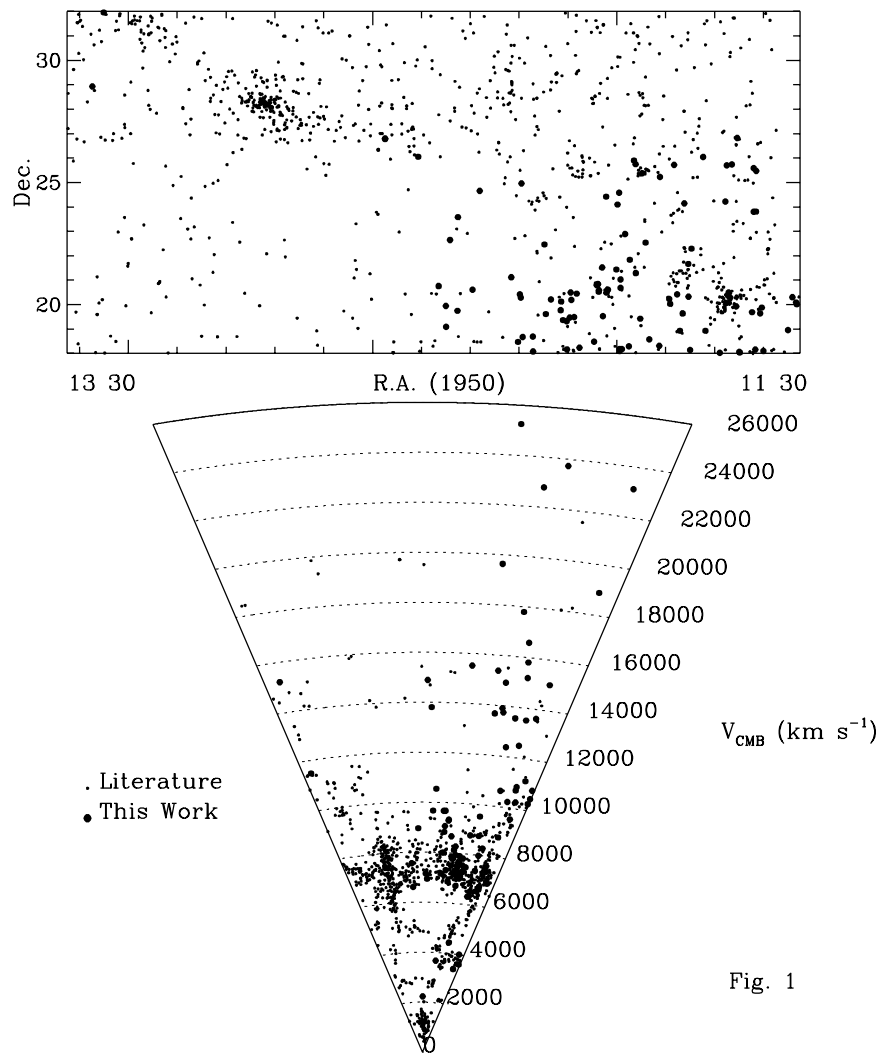

Fig. 1. The distribution in celestial coordinates of 1085 galaxies in the direction of the Coma-A 1367 supercluster, panel a). A wedge diagram ( $V_{\mathrm{CMB}}$ vs. R.A.) is given in panel b). Small symbols mark galaxies taken from the literature, filled circles mark the measurements obtained in this work

in Gavazzi 1987) (see Table 3 below for details). Figure 3 gives a representation in celestial coordinates of the foreground galaxies (including Agg = 16) panel a) and a wedge diagram ( $V_{\mathrm{CMB}}$ vs. R.A.) is given in panel b.

4) Background (Agg = 19.0): we subtract from the remaining sample all galaxies with $V>8600 \mathrm{~km} \mathrm{~s}^{-1}$, which are considered as background objects.

5) Groups $(\mathrm{Agg}=5$.$) : on the remaining sample$ $\left(6000<V<8600 \mathrm{~km} \mathrm{~s}^{-1}\right)$ we run an algorithm that counts around each galaxy the number of galaxies found within $0.9 \mathrm{Mpc}$ projected radius, satisfying the additional requirement that their velocity is within $600 \mathrm{kms}^{-1}$ from the mean velocity of the aggregate under study $\left(850 \mathrm{~km} \mathrm{~s}^{-1}\right.$ for group 5.3). If there are at least 8 such galaxies, these are assigned to the "group" class $(\mathrm{Agg}=5) .5$ groups are found by the algorithm (N3937, N4065, IC 762, N4213 and IC 3165) (see Table 3 for details). Figure 4 gives a representation in celestial coordinates of the groups members (panel a) and a wedge diagram is given in panel b. Different symbols are used for the various groups.

6) Multiplets (Agg $=6.2$ to 6.5$)$ : on the remaining sample $\left(6000<V<8600 \mathrm{~km} \mathrm{~s}^{-1}\right)$ we run an algorithm that

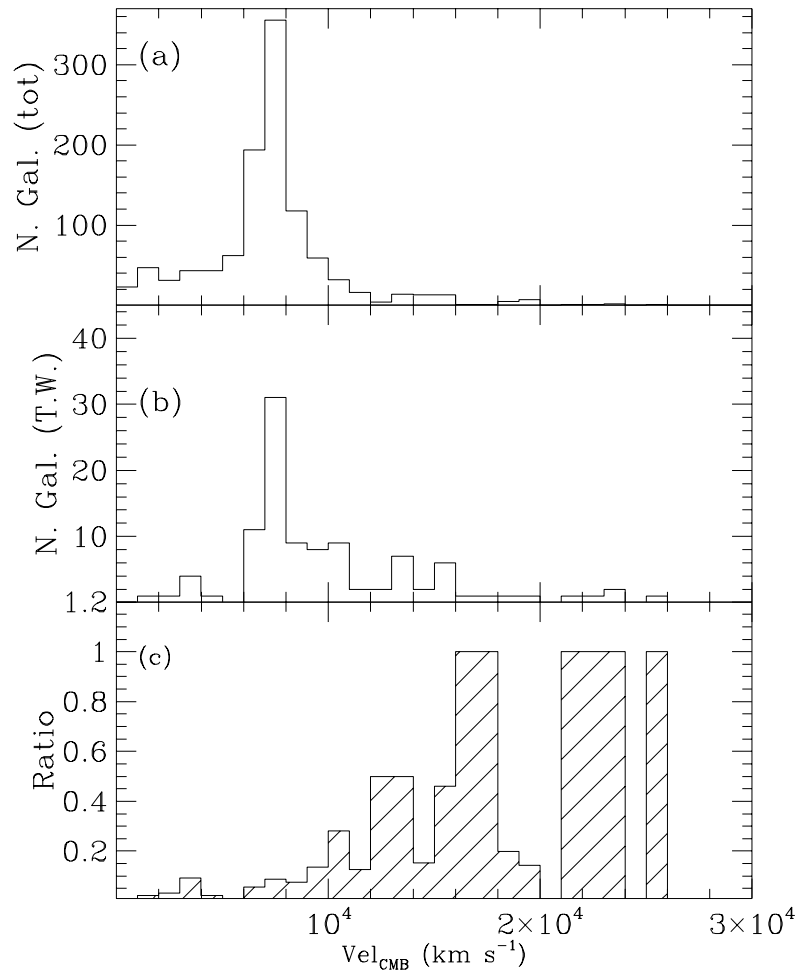

Fig. 2. Velocity histogram of the 1085 available redshifts, panel a), of the 102 redshifts obtained in this work, panel b) and of the ratio of new to all redshifts, panel c)

counts around each galaxy the number of galaxies found within $0.3 \mathrm{Mpc}$ projected radius satisfying the additional requirement that their velocity is within $600 \mathrm{~km} \mathrm{~s}^{-1}$ from the mean velocity of the aggregate under study. If there is at least 1 such galaxy, these are assigned to the "multiplet" class $(\mathrm{Agg}=6)$. $(6.2=$ doublets, $6.3=$ triplets... $6.5=$ quintuplets $)$.

7) Contact multiplets $(\mathrm{Agg}=6.1)$ : we repeat 6$)$ with a more stringent requirement that the companion galaxy lies within $50 \mathrm{kpc}$. these are contact doublets and triplets. Figure 5 gives a representation in celestial coordinates of the members to multiplets (panel a) and a wedge diagram is given in panel $\mathrm{b}$.

8) Isolated $(\mathrm{Agg}=7.0)$ : on the restricted velocity range $6000<V<8000 \mathrm{~km} \mathrm{~s}^{-1}$ we count galaxies which do not have a companion within $0.3 \mathrm{Mpc}$ projected radius. We call these isolated $(\mathrm{Agg}=7.0)$.

9) Strictly isolated $(\mathrm{Agg}=7.1)$ we repeat 8$)$ with the more stringent requirement that the galaxy under study is isolated within $0.5 \mathrm{Mpc}$ projected radius. Figure 6 gives a representation in celestial coordinates of the isolated objects (panel a) and a wedge diagram is given in panel b.

10) Extended clusters: finally we re-inject in the sample the cluster objects (see step 1) and try an alternative, less restrictive definition of cluster membership, which 
Table 3. The criteria used to define each "Aggregate" in the direction of the Coma-A 1367 supercluster

\begin{tabular}{|c|c|c|c|c|c|c|c|c|c|}
\hline$(1)$ & $\begin{array}{l}\text { Description } \\
\text { (2) }\end{array}$ & (3) & $\begin{array}{c}R / \theta \\
\mathrm{Mpc} / \mathrm{deg} . \\
(4)\end{array}$ & $\begin{array}{l}\text { R.A. } \\
\text { h m } \\
(5)\end{array}$ & $\begin{array}{l}\text { Dec. } \\
\circ, \\
(6)\end{array}$ & $\begin{array}{c}\Delta_{V} \\
\mathrm{~km} \mathrm{~s}^{-1} \\
(7)\end{array}$ & $\begin{array}{c}V_{\min }-V_{\max } \\
\mathrm{km} \mathrm{s}^{-1} \\
(8)\end{array}$ & $\begin{array}{c}<V> \\
\mathrm{km} \mathrm{s}^{-1} \\
(9)\end{array}$ & (10) \\
\hline 1.0 & Coma Core & - & $\theta<0.5$ & 1257 & 2815 & 2700 & $4000-10000$ & 7145 & 72 \\
\hline 2.0 & Coma & - & $0.5<\theta<2$ & 1257 & 2815 & 1500 & $4000-10000$ & 7244 & 105 \\
\hline & Coma ext. & $>19$ & $R<2$ & $1222-1300$ & $26-30$ & 1500 & $4000-10000$ & - & 37 \\
\hline 3.0 & A 1367 Core & - & $\theta<0.5$ & 1142 & 2006 & 2500 & $4000-10000$ & 6805 & 59 \\
\hline 4.0 & A 1367 & - & $0.5<\theta<1$ & 1142 & 2006 & 1500 & $4000-10000$ & 6967 & 22 \\
\hline & A 1367 ext. & $>19$ & $R<2$ & $1130-1155$ & $19-23$ & 1500 & $4000-10000$ & - & 40 \\
\hline 5.2 & Group N3937 & $>8$ & $R<0.9$ & 1150 & 2055 & 600 & $6000-8600$ & 7002 & 21 \\
\hline 5.3 & Group N4065 & $>8$ & $R<0.9$ & 1201 & 2030 & 850 & $6000-8600$ & 7307 & 25 \\
\hline 5.4 & Group IC762 & $>8$ & $R<0.9$ & 1205 & 2602 & 600 & $6000-8600$ & 7207 & 13 \\
\hline 5.5 & Group N4213 & $>8$ & $R<0.9$ & 1213 & 2415 & 600 & $6000-8600$ & 7054 & 10 \\
\hline 5.6 & Group IC3165 & $>8$ & $R<0.9$ & 1217 & 2815 & 600 & $6000-8600$ & 8102 & 4 \\
\hline 6.1 & Contact mult. & $>1$ & $R<0.05$ & $1130-1330$ & $18-32$ & 600 & $6000-8600$ & - & 26 \\
\hline 6.2 & Doublets & 1 & $R<0.3$ & $1130-1330$ & $18-32$ & 600 & $6000-8600$ & - & 55 \\
\hline 6.3 & Triplets & 2 & $R<0.3$ & $1130-1330$ & $18-32$ & 600 & $6000-8600$ & - & 23 \\
\hline 6.4 & Quadruplets & 3 & $R<0.3$ & $1130-1330$ & $18-32$ & 600 & $6000-8600$ & - & 9 \\
\hline 6.5 & Quintuplets & 4 & $R<0.3$ & $1130-1330$ & $18-32$ & 600 & $6000-8600$ & - & 1 \\
\hline 7.0 & Isolated & 0 & $R<0.3$ & $1130-1330$ & $18-32$ & - & $6000-8000$ & - & 75 \\
\hline 7.1 & Stric. Isolated & 0 & $R<0.5$ & $1130-1330$ & $18-32$ & - & $6000-8000$ & - & 122 \\
\hline 19.0 & Background & - & - & $1130-1330$ & $18-32$ & - & $8000-$ & - & 219 \\
\hline 8.0 & Foreg. Gr. N3798 & - & - & $1130-1143$ & $24-32$ & - & $3000-4000$ & 3883 & 5 \\
\hline 9.0 & Foreg. Gr. N3801 & - & - & $1130-1143$ & $18-24$ & - & $3000-4000$ & 3671 & 13 \\
\hline 10.0 & Foreg. Gr. N3995 & - & - & $1143-1209$ & $18-32$ & - & $3000-4000$ & 3691 & 21 \\
\hline 11.0 & Foreg. Gr. N4005 & - & - & $1145-1200$ & $22-32$ & - & $4000-5500$ & 4864 & 20 \\
\hline 12.0 & Foreg. Gr. IC202 & - & - & $1146-1214$ & $18-22$ & - & $4500-5500$ & 4803 & 3 \\
\hline 13.0 & Foreg. Gr. N4169 & - & - & $1200-1220$ & $26-32$ & - & $3500-4500$ & 4210 & 13 \\
\hline 14.0 & Foreg. Gr. N4615 & - & - & $1214-1250$ & $24-32$ & - & $4500-5700$ & 4996 & 14 \\
\hline 15.0 & Foreg. Gr. U8248 & - & - & $1303-1330$ & $18-26$ & - & $3500-4500$ & 4057 & 7 \\
\hline 16.0 & Foreg. Gr. N5056 & - & - & $1303-1330$ & $28-32$ & - & $5000-6700$ & 5885 & 25 \\
\hline 17.0 & Foreg. Gr. N4794 & - & - & $1130-1330$ & $18-32$ & - & $2300-3200$ & 2813 & 24 \\
\hline 18.0 & Foreg. Gr. C1cl. & - & - & $1130-1330$ & $18-32$ & - & $0-2200$ & 1237 & 79 \\
\hline 19.1 & $z=0$ & - & - & $1130-1330$ & $18-32$ & - & -- & - & 45 \\
\hline
\end{tabular}

includes more peripheral objects. We count around each galaxy, in the interval $4000<V<10000 \mathrm{~km} \mathrm{~s}^{-1}$, the number of galaxies found within $2.0 \mathrm{Mpc}$ projected radius. Within $2<R<8.5 \mathrm{deg}$ of the center of Coma and $1<R<3.5 \mathrm{deg}$ of the center of $\mathrm{A} 367$ we allow for a velocity difference of $1500 \mathrm{~km} \mathrm{~s}^{-1}$ from the mean velocity of the cluster. In both aggregates we consider a galaxy to belong to the "extended cluster" if there are $>20$ galaxies satisfying the above criterion. Figure 7 gives a representation in celestial coordinates of the cluster members (panel a) and a wedge diagram is given in panel b. Different symbols are used for the core, outskirt and extended cluster members.

Figure 8 gives a representation in celestial coordinates of the supercluster members (panel a) and a wedge diagram is given in panel $b$.

All the above aggregation classes (1-19) are mutually exclusive, except the definition of extended cluster. A galaxy belonging to the extended cluster can be previously defined as belonging to a group, multiplet or even be considered isolated.

The criteria used above are summarized in Table 3 as follows: For any Agg class (Col. 1) a decription is given in Col. 2.

Column 3: the minimum number of objects used to compute the threshold density of each aggregate.

Column 4: the scale ( $R$ in Mpc; $\theta$ in degrees) used to compute the threshold density of each aggregate.

Columns 5, 6: the "window" in celestial coordinates where each aggregate was found. A single coordinate gives the center of the aggregate (with radius $R$ or $\theta$ ). If a pair of coordinates is given this indicates the interval where the aggregate was found.

Column 7: the allowed velocity difference from the average velocity of the aggregate.

Columns 8, 9: the velocity interval where the aggregate was found.

Column 10: the average velocity of the aggregate. 

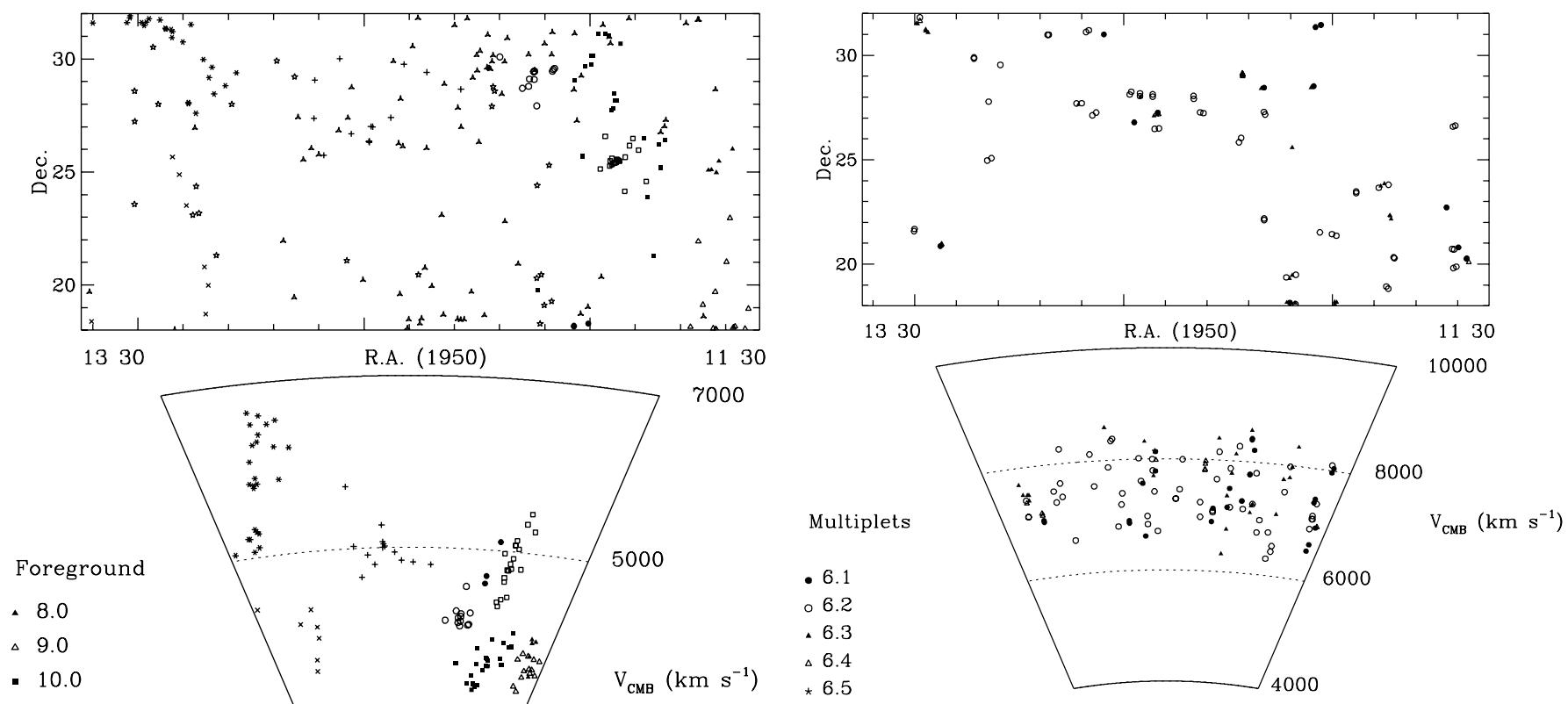

Fig. 5

Fig. 5. The distribution in celestial coordinates of galaxies belonging to multiplets $(\mathrm{Agg}=6)$ in the Coma-A 1367 superFig. 3 cluster, panel a). A wedge diagram is given in panel b)

Fig. 3. The distribution in celestial coordinates of galaxies in the foreground (Agg = 8-18) of the Coma-A 1367 supercluster, panel a). A wedge diagram is given in panel $\mathbf{b}$ )

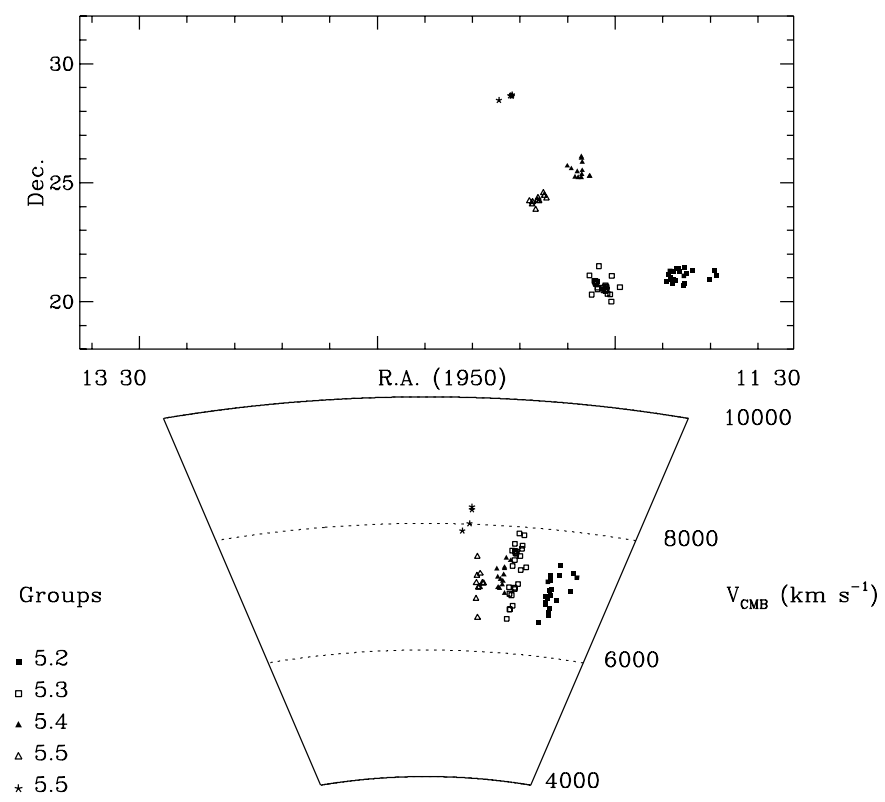

Fig. 4

Fig. 4. The distribution in celestial coordinates of galaxies belonging to 5 groups $(\mathrm{Agg}=5)$ in the Coma-A 1367 supercluster, panel a). A wedge diagram is given in panel $\mathbf{b}$ )

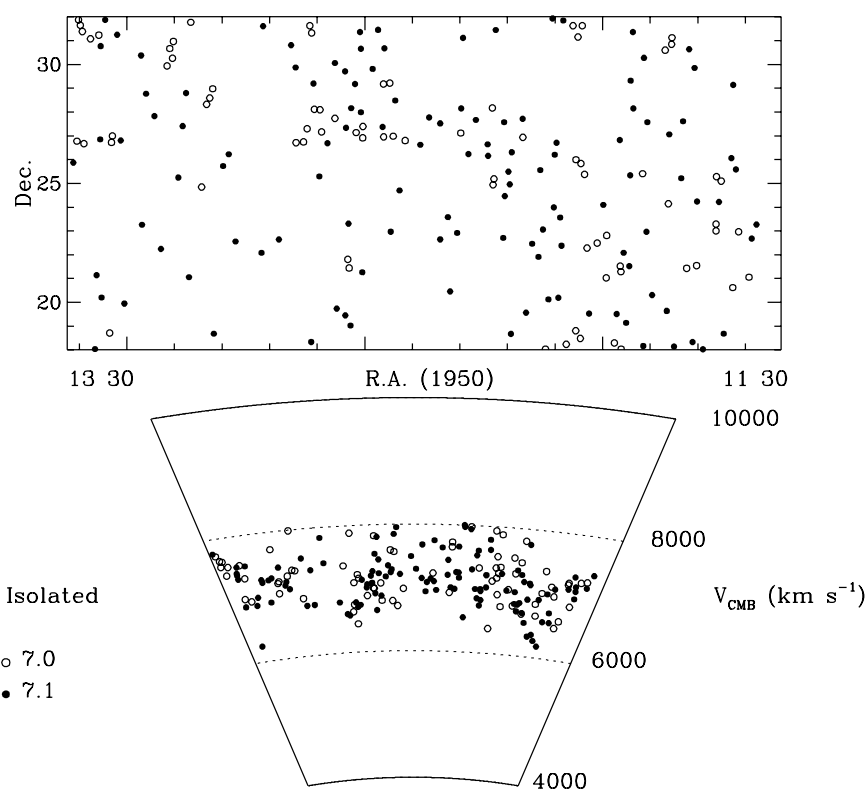

Fig. 6

Fig. 6. The distribution in celestial coordinates of isolated galaxies $(\mathrm{Agg}=7)$ in the Coma-A 1367 supercluster, panel a). A wedge diagram is given in panel $\mathbf{b}$ ) 


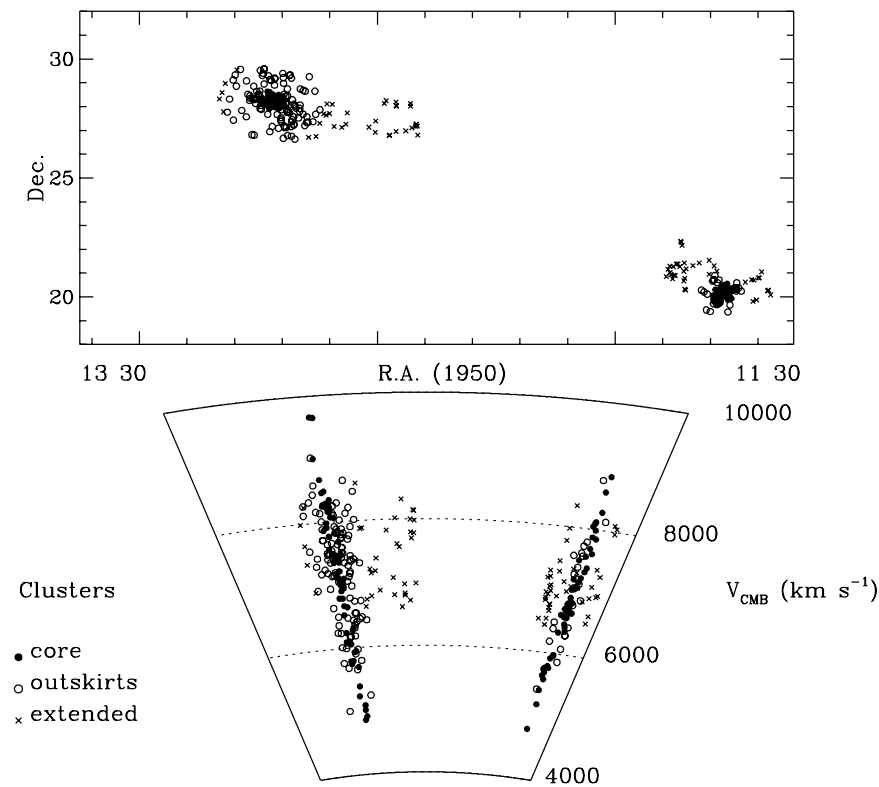

Fig. 7

Fig. 7. The distribution in celestial coordinates of galaxies belonging to the Coma and A 1367 clusters (Agg =1-4), panel a). A wedge diagram is given in panel $\mathbf{b}$ )

Column 11: the number of objects belonging to each aggregate.

\section{Conclusions and summary}

We obtained 117 new redshift measurements of GCGC galaxies, 102 of which in the direction of the ComaA 1367 supercluster. With the new data, the total number of available redshifts in this direction of the sky is 1068 $\left(m_{\mathrm{p}} \leq 15.7\right)$.

The supercluster is centered at $\sim 7200 \mathrm{~km} \mathrm{~s}^{-1}$ and it extends from 6000 to $8500 \mathrm{~km} \mathrm{~s}^{-1}$. It contains two rich clusters (Coma + A 1367) whose "fingers of God" stretch from 4000 to $10000 \mathrm{~km} \mathrm{~s}^{-1}$.

By computing of the local galaxy density around each object, we separate galaxies belonging to five groups, from several multiplets and relatively isolated objects within the supercluster. This classification will be used to study the environmental dependence of properties of galaxies spanning over an order of magnitude in local density.

Acknowledgements. We wish to thank the TACS of the Loiano and Cananea telescopes for the generous amounts of time

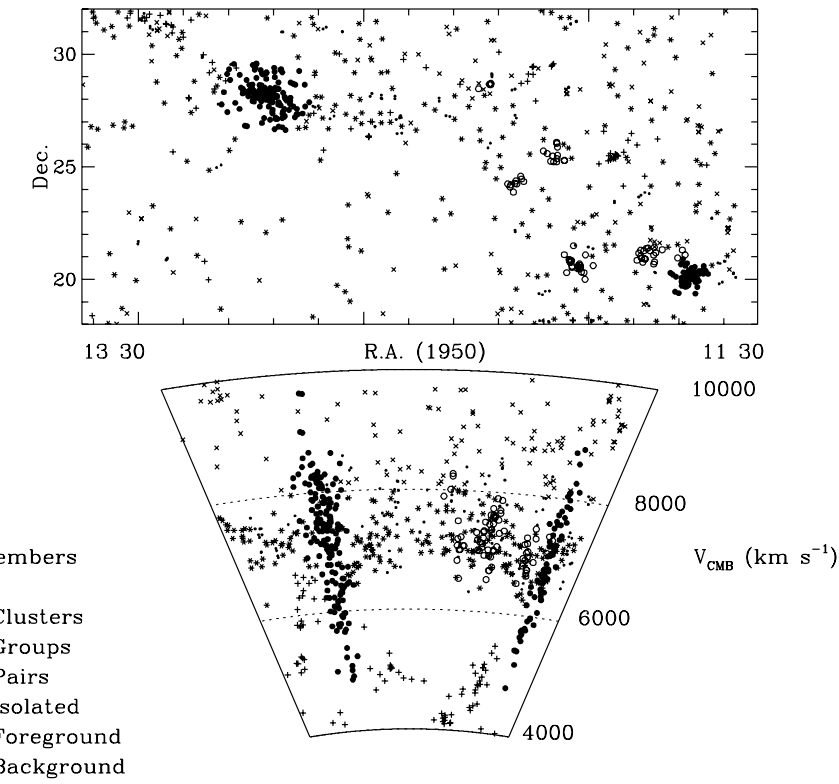

Fig. 8

Fig. 8. The distribution in celestial coordinates of members $($ Agg $=1-7)$ to the Coma-A 1367 supercluster, panel a). A wedge diagram is given in panel $\mathbf{b}$ )

allocated to this project. G.G. wishes to thank the students of his course for their contribution during the observations and the data reduction. L.C. has had support from CONACYT (México) research grant No. 211290-5-1430PE.

\section{References}

de Lapparent V., Geller M., Huchra J., 1986, ApJ 302, L1

Gavazzi G., ApJ 320, 96

Gavazzi G., Randone I., Branchini E., 1995, ApJ 438, 590

Gavazzi G., Boselli A., 1996, Astroph. Lett. Comm. 35, 1

Giovanelli R., Haynes M., 1985, ApJ 292, 404

Gregory S., Thompson L., 1978, ApJ 222, 784

Huchra J., Geller M., de Lapparent L., Corwin H., 1990, ApJS 72,433

Kogut A., et al., 1993, ApJ 419, 1

Nilson P., 1973, Uppsala Obs. Ann. 6 (UGC)

Tonry J., Davis M., 1979, AJ 84, 1511

Zabludoff A., Geller M., Huchra J., Ramella M., 1993, AJ 106, 1301

Zwicky F., Herzog E., Karpowicz M., Koval C., Wild P., 1961-1968, Catalogue of Galaxies and Clusters of Galaxies. Pasadena: Caltech (GCGC) 